attacks per day. With medical therapy, physical and speech therapies were regularly performed. Because of progression of the disease and poor response to therapy, surgical treatment was proposed. In September 2018, left functional hemispherotomy was performed at the clinic of neursciences in Paris, after which the convulsions completely disappeared. From all of the above, we conclude that functional hemispherotomy is a therapy of choice in the treatment of resistant attacks in Rasmussen's syndrome, which stops progression of the disease and prevents further neurological damage.

\section{P502 YERSINIA ENTEROLITICA INFECTION WITH ENCEPHALOPATHY}

laurent Pradeaux*, Nabil Bendjazia, marie Percot-Blondy, loic Fritsch, maud Sordet, sandra Pochelu, daniela Behrendt. Centre Hospitalier, Périgueux, France

10.1136/archdischild-2019-epa.838

We want to describe a 8 year old boy with no medical problem before. The boy came at the emergency department at the night with general weakness, hallucinations and difficulties to walk.

When he arrived the temperature was $37,1^{\circ}$, glycemia 6,6 $\mathrm{mmol} / \mathrm{l}$. He had normal vital signs, a pulse rate of 95 beats per minute and a normal blood pressure $(107 / 53 \mathrm{mmHg}$ ). $\mathrm{He}$ was lethargic, had hallucinations and he had a cerebellar ataxia. Pupils were dilated but reactive. Reflexes were normal. On admission standard blood test (cell count, hepatic parameter, C-reactive protein) was normal. Urine and serum toxicology screen was negative. Cerebral fluid examination revealed no white cell and a normal glucose, protein concentration. The electroencephalogram showed left temporal slow waves. Brain magnetic resonance imaging (MRI) was normal. Herpes simplex virus encephalitis was suspected and aciclovir was started. Three days later the aciclovir was stopped (PCR herpes negative), PCR enterovirus as well.

At the stool culture, we found a yersinia enterocolitica, biotype 4, serotype 0:3. This pathogen agent was the only one we found. We began an antibiotic treatment when we got the result but the boy's clinical state improved before with no more ataxia at day two of hospitalisation. The hallucinations persisted during four days. The boy was discharged at day eight. Four weeks later the electroencephalogram and MRI were normal. Clinical examination was perfect.

Yersinia enterolitica is a common cause of acute gastroenteritis in our countries. The contamination by fecal-oral route, invasive forms (abscesses, septicemia) occurs in patients with underlying conditions (cirrhosis, diabetes). Secondary complications such as reactive arthritis or erythema nodosum are known. An acute or chronic neurological disease is possible but rare.

On a follow up of 458 hospitalized patients in Norway 14 presented neurological manifestations. The different expressions were headache, dizziness, polyradiculitis. Another report of an 8 years old girl appeared similar to our present case (1). Yersinia enterolitica is not know to secrete a neurotoxin. Yersinia enterolitica secrete a heat stable enterotoxin and can induce immunologic response. Mechanism of acute or chronic neurological manifestations remain unclear but must not be disregard these and could induce a field of search (2).

\section{REFERENCES}

1. Berner R, Kist M, Sauer M. Encephalopathy associated with yersinia enterolitica. The Lancet 1998; $351: 418$

2. Bancerz-kisiel A, Pieczywek M, Lada P, Szweda W. The most important virulence markers of yersinia enterolitica and the role during infection. Genes $2018 ; 9$ :235-249.

\section{P503 AN EYE-OPENING CASE ANISCORIA}

${ }^{1,2}$ Elinor Jenkins*, ${ }^{2,3}$ David W Webb, ${ }^{2}$ Mary 0 Regan. ${ }^{1}$ Trinity College Dublin, School of Medicine, Dublin , Ireland; ' ${ }^{2}$ Our Lady's Hospital for Sick Children , Dublin , Ireland; ${ }^{3}$ Trinity College Dublin, School of Medicine, Dublin, Ireland

\subsection{6/archdischild-2019-epa.839}

Introduction Aniscoria is a condition characterised by pupils of unequal size. Aniscoria may be completely benign and physiological. However it can also be associated with serious lifethreatening conditions and therefore prompts alarm in clinicians.

Case Description A 4-year-old boy referred by his family doctor to hospital with a sudden onset dilated and un-reactive left pupil.

His mother had noticed the abnormal pupil on waking that morning, approximately 6 hours earlier. Her son did not appear to be in pain and his vision was unaffected. He appeared to be rubbing his eye at times but his awareness and behaviour were unaltered. There was no history of trauma and he was systemically well.

Background This little boy had a known diagnosis of Creatine Transporter Deficiency, an inherited metabolic disorder associated with learning disability and epilepsy. Physically he was mobile and very active with a mild torticollis, recurrent epistaxis and a tendency to salivate excessively. There was no recent history of headache, nausea or change in seizures. Medications at the time of presentation included Epilim Chrono (450 mg nocte), Flixotide $(125 \mathrm{mcg})$ and a Scopoderm patch.

Examination confirmed a fixed dilated left pupil with a left esotropia but otherwise normal eye movement, no ptosis and no obvious conjunctival injection. His neurological exam revealed no altered awareness and no motor deficit.

Discussion Aniscoria is common - found in approximately $20 \%$ of children (Fierz, F et al 2017). However the asymmetry is mild and pupil reactive. Aniscoria of $>1.3 \mathrm{~mm}$ is unlikely to be physiologic in children and warrants careful assessment (Suh et al 2016).

In the absence of a third nerve lesion or trauma to the eye, application of a pharmacological agent with mydriatic effect is the most likely explanation in childhood. Scopoderm patches have been reported as causing aniscoria in a 30-yearold nurse (Shah et al, 2017) and a 14-year-old boy (Lin, 2001). A recent article explored the use of hyoscine patches to reduce drooling in children with neurodisability (Parr et al, 2017) citing aniscoria as one of a number of side-effects when compared to glycopyrronium liquid.

Conclusion Following discussion with his mother involvement of his scopoderm patch was considered to be the most likely cause of his dilated pupil and no further investigations were felt necessary. His mydriasis fully resolved over three days. Children with a neuro-disability can be extremely active -making it hard to keep medication out of their way! 